\title{
Chloroquine induces apoptosis in pancreatic neuroendocrine neoplasms via endoplasmic reticulum stress
}

\author{
Kenzo Nakano, Toshihiko Masui ${ }^{D}$, Akitada Yogo, Yuichiro Uchida, Asahi Sato, Yosuke Kasai, Kazuyuki Nagai, \\ Takayuki Anazawa, Yoshiya Kawaguchi and Shinji Uemoto
}

Department of Surgery, Kyoto University Graduate School of Medicine, Kyoto, Japan

Correspondence should be addressed to T Masui: tmasui@kuhp.kyoto-u.ac.jp

\begin{abstract}
Although pancreatic neuroendocrine neoplasms (PanNENs) are generally indolent, patients with distant metastasis have a dismal prognosis. Recently, the autophagy inhibitor chloroquine (CQ) has been shown to suppress the tumour growth of PanNENs, but the detailed mechanisms have not been elucidated. Furthermore, these results were obtained from poorly differentiated cell lines rather than well-differentiated cell lines, which is the most prevalent type in this tumour. To explore the mechanism and efficacy of CQ on PanNENs, we applied CQ to cell lines and evaluated the resulting apoptosis and endoplasmic reticulum (ER) stress. CQ treatment induced ER stress, and an unfolded protein response was activated through the PERK-elF2 $\alpha$-ATF4 pathway, resulting in the expression of the pro-apoptotic protein C/EBP homologous protein (CHOP), which reflects ER-stress-mediated apoptotic cell death. Furthermore, hydroxychloroquine (HCQ) was effective in Men1 heterozygous-deficient (Men 1+/LN3-8) mice, a mouse PanNEN model that is considered to correspond to human low-grade PanNEN. HCQ administration decreased tumour size in Men1+/AN3-8 mice. In the HCQ group, histological analyses revealed that proliferative activity was unchanged, but apoptosis was accelerated, accompanied by $\mathrm{CHOP}$ expression. These results suggest that autophagy inhibition by CQ/HCQ could be used for the treatment of PanNEN, including the well-differentiated type.
\end{abstract}

Key Words

- pancreatic neuroendocrine neoplasm

- autophagy

- multiple endocrine neoplasia

- endoplasmic reticulum stress

- chloroquine

\section{Introduction}

Pancreatic neuroendocrine neoplasms (PanNENs) comprise approximately $1-2 \%$ of all pancreatic neoplasms (Yao et al. 2008). The 2017 World Health Organization (WHO) classification divides PanNENs into well-differentiated pancreatic neuroendocrine tumours (PanNETs) and poorly differentiated pancreatic neuroendocrine carcinoma (PanNEC). Although pancreatic adenocarcinoma (PDAC) has a very dismal clinical prognosis, PanNENs have been regarded as relatively indolent. However, even welldifferentiated PanNETs have the malignant potential to metastasise to distant organs, with a 5-year survival rate limited to approximately 50\% (Dasari et al. 2017). Although recent therapeutic advances using hormonal, cytotoxic, and molecular-targeted drugs have improved the long-term survival of PanNEN patients (Lee et al. 2015), the progression-free survival for each drug is limited to approximately 1 year and a variety of therapeutic options from different avenues are inevitably required.

Autophagy is a lysosomal degenerative pathway of organelles or foreign proteins that supplies amino acids (c) 2020 Society for Endocrinology Published by Bioscientifica Ltd. Printed in Great Britain 
to meet the metabolic demands of cells (Klionsky \& Emr 2000). Thus, the inhibition of autophagy is a potential target for treating malignant tumours. Indeed, the autophagy inhibitor chloroquine (CQ) and its derivative hydroxychloroquine (HCQ) have been tested against various types of cancer, including lung, colorectal, and pancreatic cancers (Chude \& Amaravadi 2017, Xu et al. 2018). Moreover, Avniel-Polak et al. reported that CQ induced apoptosis in the BON-1 PanNEN cell line in vitro (Avniel-Polak et al. 2016) and in a subcutaneous xenograft model (Avniel-Polak et al. 2018). CQ inhibits the activity of lysosomal enzymes in autophagolysosomes resulting in the accumulation of autophagolysosomes in the cytoplasm. However, the precise mechanisms underlying the effects of CQ in PanNEN remain poorly understood. In addition, these models are all derived from poorly differentiated PanNEC, which has a different genetic status compared with well-differentiated PanNET.

In this study, we applied the autophagy inhibitor CQ to the MIN6 and QGP-1 PanNEN cell lines and HCQ to genetically engineered Men $1^{+/ \Delta N 3-8}$ PanNET mice to clarify the cytotoxic effects of HCQ. We also identified one of the mechanisms of this cytotoxic effect in these tumours.

\section{Materials and methods}

\section{Cell lines}

MIN6 cells, derived from mouse insulinomas induced by insulin promoter-driven SV40 T antigens, which supresses p53 and RB activity (Miyazaki et al. 1990), were kindly donated by Dr Jun-ichi Miyazaki (Graduate School of Medicine, Osaka University, Osaka, Japan). The culture medium was high-glucose Dulbecco's modified Eagle medium (DMEM) supplemented with 10\% foetal bovine serum (FBS) and 1\% penicillin/streptomycin. The cells were stocked at a passage number of 12 to 16 and utilised at 20 to 40 passages. QGP-1 cells, which were established from human malignant pancreatic somatostatinomas (Kaku et al. 1980, Iguchi et al. 1990), were purchased from the Japan Cancer Research Resources Bank Cell Bank, Osaka, Japan. According to the COSMIC Cell Line Project (https://cancer.sanger.ac.uk/cell_lines, COSMIC sample ID: COSS1298534), QGP-1 cells have heterozygous mutations in KRAS (G12D) and TP53. The culture medium used was RPMI1640 (Nacalai Tesque, Kyoto, Japan) supplemented with 10\% FBS and 1\% penicillin/ streptomycin. The cells were stocked at a passage number of 7 and utilised at 10 to 25 passages. Both cell lines were cultured at $37^{\circ} \mathrm{C}$ in a cell incubator with $5 \% \mathrm{CO}_{2}$ and were passaged $1: 6$ or $1: 8$ after achieving $>90 \%$ confluence. To induce autophagy, the cells were incubated with highglucose DMEM without pyruvate (048-33575; FUJIFILM Wako Pure Chemical Corporation). In the cell growth assay, $2.0 \times 10^{5}$ cells/well were plated into 24 -well plates, incubated for 3 days, and then supplemented with the given compounds. Cell counts were obtained at the time of treatment initiation and at given times in triplicate using the Countess Automated Cell Counter ${ }^{\mathrm{TM}}$ (Invitrogen). Growth rates were calculated based on these cell counts. For the immunofluorescence analysis, the cells were fixed via incubation with $4 \%$ paraformaldehyde (PFA) for $20 \mathrm{~min}$, followed by being washed with PBS, and then incubated with $0.5 \%$ Triton-X 100/PBS for 20 min. After another washing with PBS and blocking with Protein Block Serum-Free Ready-to-use (Agilent) for $30 \mathrm{~min}$, the cells were incubated with the primary antibody overnight at $4^{\circ} \mathrm{C}$. After three washes with PBS, the cells were incubated with the secondary antibody for $30 \mathrm{~min}$ at $4^{\circ} \mathrm{C}$ and observed under fluorescent microscopy. The primary and secondary antibodies used are listed in Supplementary Table 1 (see section on supplementary materials given at the end of this article). The cell count data were calculated by analysing three to six high-power fields (HPFs) for each group.

\section{Transmission electron microscopy (TEM)}

To perform the TEM of cell lines, 4\% PFA and 2\% glutaraldehyde (GA)/PBS were added to the culture dish of cell lines. The cells were incubated overnight at $4^{\circ} \mathrm{C}$ and brought to the Division of Electron Microscopic Study (DEMS) in the Center for Anatomical Studies, Graduate School of Medicine, Kyoto University. DEMS conducted the osmium tetroxide post-fixation and the preparation of ultrathin sections with uranium staining. The sections were then observed under TEM (Hitachi H-7650; Hitachi).

\section{RNA isolation and data analysis}

Total RNA was extracted using the RNeasy Mini Kit (Qiagen) in accordance with the manufacturer's protocols. First-strand cDNA synthesis was performed using the ReverTra Ace qPCR RT Master Mix (Toyobo, Osaka, Japan). RT-PCR was used to measure the ratio of spliced to total Xbp1/XBP1 mRNA. The forward primer 5'-AGTTAAGAACACGCTTGGGAAT-3' and the reverse primer 5'-AAGATGTTCTGGGGAGGTGAC-3' were used for the MIN6 cells originating from the mice. 
The resulting PCR products were $172 \mathrm{bp}$ in length for the unspliced Xbp1 mRNA and $146 \mathrm{bp}$ in length for the spliced Xbp1 mRNA. The forward primer 5'-AATGAAGTGAGGCCAGTGGCC-3' and the reverse primer 5'-AATACCGCCAGAATCCATGGG-3' were used for the QGP-1 cells derived from human tumours. The resulting PCR products were $125 \mathrm{bp}$ in length for the unspliced XBP1 mRNA and $99 \mathrm{bp}$ in length for the spliced XBP1 mRNA. The products were electrophoresed in $10 \%$ polyacrylamide gel in TBE, stained with ethidium bromide, and visualised using the ChemiDoc XRS+ system (Bio-Rad Laboratories). The intensity of the bands was quantified using Image Lab Software (Bio-Rad Laboratories), and the intensity ratio was calculated.

\section{Protein lysates and Western blot analysis}

Whole-cell lysates were prepared by adding RIPA buffer supplemented with protease inhibitor (cOmplete Mini; Roche). Protein yield was calculated using the BCA assay. Total protein lysates were denatured in SDS sample buffer separated by SDS-PAGE and transferred to PVDF membranes. The membranes were blocked and then incubated with the primary antibody in 5\% skim milk and $0.5 \%$ Tween 20/Tris-buffered saline (TBST) overnight at $4^{\circ} \mathrm{C}$. After three washes in TBST, the membrane was incubated with the secondary antibody (5\% skim milk in TBST) for $1 \mathrm{~h}$ at room temperature. The primary/ secondary antibodies we used are listed in Supplementary Table 2. Chemiluminescence was detected with ChemiLumi One Super (Nacalai Tesque) and visualised with the ImageQuant LAS 4000 (GE Healthcare). The intensity of the bands was quantified using ImageQuant TL software (GE Healthcare), and the intensity ratio was calculated. To assess the systemic inhibition of autophagy, wild-type mice $(n=2)$ were administered $10 \mathrm{mg} / \mathrm{kg}$ of HCQ for 7 consecutive days. Tissue samples were collected from the ear and tail before and after treatment. Proteins from each tissue sample were analysed.

\section{Mice}

FVB;129S-Men1 ${ }^{\text {tm1.1Ctre }} / \mathrm{J}$ mice (hereinafter referred to as Men $1^{+/ \Delta N 3-8}$ mice) were purchased from Jackson Laboratory (stock no. 004066). Eighteen-month-old mice were intraperitoneally injected with HCQ $(10 \mathrm{mg} / \mathrm{kg})$ or saline. The mice were killed after 21 consecutive daily injections. The pancreas and other tumour-bearing organs were collected, and histological and pathological analyses were performed. We dissected the whole pancreas at $100-\mu \mathrm{m}$ intervals and measured the diameters of all islets (usually $<200 \mu \mathrm{m}$; large islets, 200-500 $\mu \mathrm{m}$ ) or tumours ( $>500 \mu \mathrm{m}$ ) on the single section displaying the maximum tumour area. All procedures were approved by the Kyoto University Ethics Committee for Animal Experiments.

\section{Immunohistochemistry and immunofluorescence}

Paraffin sections were cut into 4- $\mu$ m-thick slices, deparaffinised, and rehydrated. For the immunohistochemistry and immunofluorescence analyses, the procedures from antigen retrieval to antibody reaction were performed as previously reported (Furuyama et al. 2011). The primary and secondary antibodies used are listed in Supplementary Table 1. The slices were treated with $\mathrm{DAB}$ peroxidase substrate (SK-4100; Vector Laboratories) for immunohistochemistry or with the TSA Plus System (PerkinElmer) and DAPI for immunofluorescence. For the TdT-mediated dUTP nick end labelling (TUNEL) assays, the DeadEnd Fluorometric Apoptosis Detection System (Promega Corporation) was used in accordance with the manufacturer's protocols.

\section{Statistical analysis}

All values are shown as the mean \pm s.D. All error bars represent the s.D. All indices were analysed using an unpaired $t$-test (two-tailed). $P$-values $<0.05$ were considered significant. The statistical analyses were performed using JMP Pro version 14.0 software (SAS Institute).

\section{Results}

\section{CQ treatment suppressed autophagy and growth of PanNEN cells}

First, we treated the PanNEN cell line MIN6 with CQ. As expected, the TEM analyses showed the chaotic accumulation of autophagolysosomes in the cytoplasm (Fig. 1A, yellow arrowheads), which supports previous reports of the inhibitory effect of CQ on lysosomal enzymes (Klionsky et al. 2016). Accordingly, LC3B-II protein accumulation was evident in the Western blot analyses, which supports the accumulation of autophagolysosomes (Fig. 1B). CQ-treated QGP-1 cells displayed similar findings (Fig. 1C and D). These data indicate that CQ treatment suppressed autophagy in PanNEN cells.

The MIN6 cells showed a significantly reduced growth rate with CQ treatment (Fig. 2A). Cytologically, CQ 

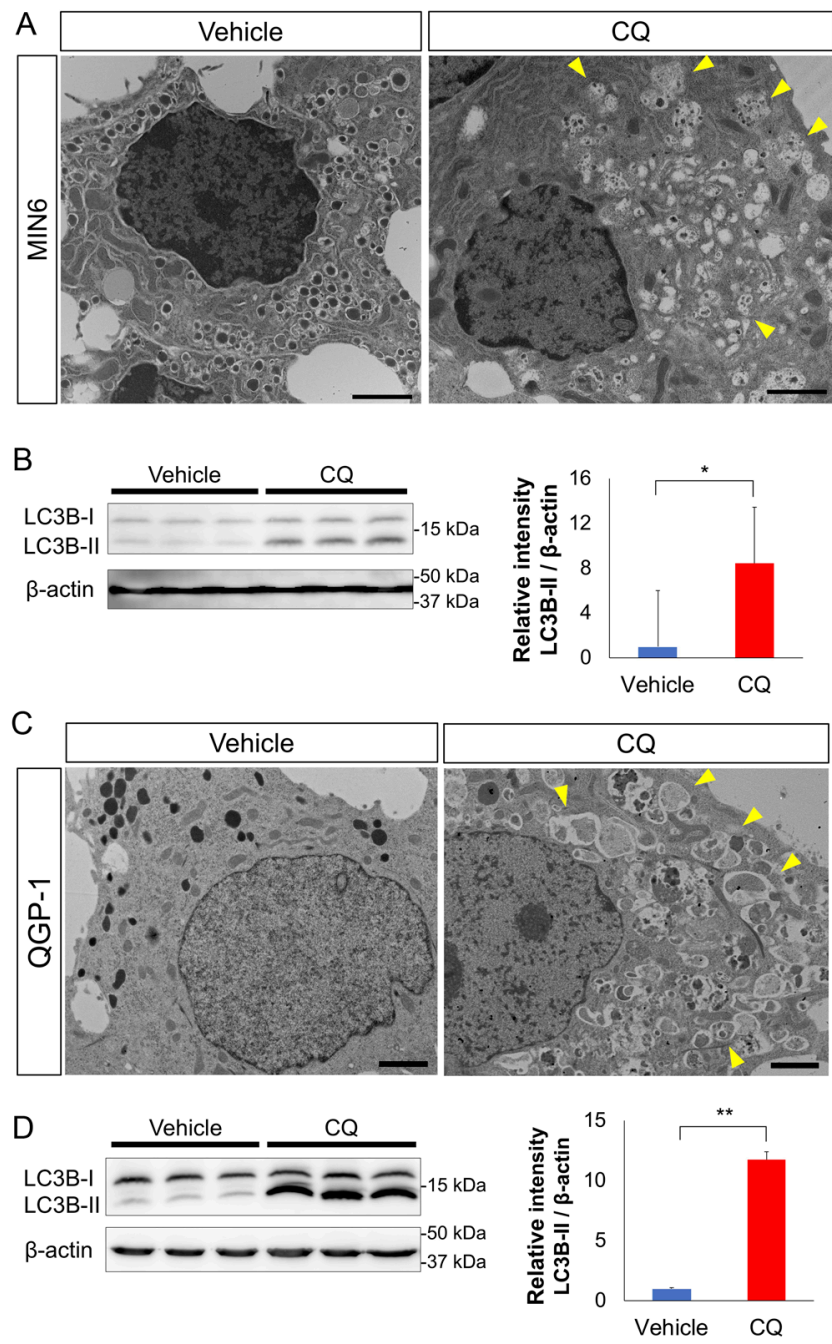

\section{Figure 1}

Analyses of the effects of CQ on MIN6/QGP-1 cells. (A) TEM analyses of MIN6 cells supplemented with a vehicle (left panel) or CQ (right panel). CQ: $20 \mu \mathrm{M}$. Yellow arrowheads: autophagolysosomes. Bar: $2 \mu \mathrm{m}$. (B) Western blot analysis of cell lysates from MIN6 cells for LC3. The relative intensity of the bands ( $n=3$ in each group) is shown on the right. (C) TEM analyses of QGP-1 cells supplemented with a vehicle (left panel) or CQ (right panel). CQ: $200 \mu \mathrm{M}$. Bar: $2 \mu \mathrm{m}$. (D) Western blot analysis of cell lysates from QGP-1 cells for LC3. The relative intensity of the bands ( $n=3$ in each group) is shown on the right.

treatment increased the proportion of cleaved caspase3-positive cells and decreased the proportion of Ki-67positive cells, which indicates activated apoptosis and suppressed proliferation, respectively (Fig. $2 \mathrm{~B}$ and $\mathrm{C}$ ). Similar results were shown in the QGP-1 cells upon CQ treatment (Fig. 2D, E and F).

\section{CQ induced ER stress-mediated apoptosis}

During our TEM analyses, we noticed that the lumen of the ER was dilated by CQ treatment in the MIN6 cells, this response was similar to the results of treatment with tunicamycin, a well-known inducer of ER stress (Fig. 3A). Based on extensive studies of the interaction between autophagy and ER stress and ER-stress-mediated apoptosis (Ogata et al. 2006, Ron \& Walter 2007, Tabas \& Ron 2011), we hypothesised that ER stress represented the trigger of CQ-induced apoptosis in PanNEN cells. This finding prompted us to examine the three major unfolded protein response (UPR) pathways (Yoshida et al. 2000, Ron \& Walter 2007). In the MIN6 cells, increased ATF4 expression was observed with CQ treatment (Fig. 3B), whereas the cleavage of ATF6 remained unchanged (Fig. 3C). The IRE1 pathway was not activated because the splicing of X-box-binding protein 1 (Xbp1) mRNA was not accelerated (Fig. 3D). The immunofluorescent analyses showed that a subset of ATF4-positive cells expressed C/EBP homologous protein (CHOP), a proapoptotic protein (Fig. 3E). Moreover, the expression of cleaved caspase-3 was predominantly detected in CHOP-positive cells (Fig. 3F). These findings indicate that the PERK-eIF2 $\alpha$-ATF 4 pathway was activated by CQ treatment and that excess UPR caused apoptosis through the induction of CHOP in the MIN6 cells. Similar TEM findings were observed in the QGP-1 cells (Fig. 4A). We confirmed the upregulation of the PERK-eIF2 $\alpha$-ATF4-CHOP pathway in QGP-1 cells (Fig. 4B). Again, no significant difference in ATF6 cleavage was identified (Fig. 4C). Unlike the MIN6 cells, the QGP-1 cells displayed accelerated splicing of XBP1 mRNA (Fig. 4D). We also noted that the expression of cleaved caspase- 3 was predominantly detected in CHOP-positive QGP-1 cells (Fig. 4E).

\section{HCQ treatment induced apoptosis and reduced tumour size in Men1+/LN3-8 mice}

We used Men $1^{+/ \Delta N 3-8}$ mice to validate the effect of an autophagy inhibitor on PanNEN in vivo. Men $1^{+/ \Delta N 3-8}$ mice reportedly develop pancreatic islet tumours with approximately $80 \%$ penetrance by 16 months of age (Crabtree et al. 2001). Among the 30 mice analysed, we found that all mice bore visible tumours (diameter $>1 \mathrm{~mm}$ ) in the pancreas at 18 months (Supplementary Fig. 1A). As previously reported (Bertolino et al. 2003, Loffler et al. 2007), the islet tumours in the Men $1^{+/ \Delta N 3-8}$ mice consisted of chromogranin A-positive cells without severe atypia; moreover, they were highly vascularised, sharing the characteristics of human well-differentiated PanNETs (Supplementary Fig. 1B and C). We found that the Ki-67 labelling index was $<3 \%$, which is comparable to human NET Grade 1 (G1) according to the 2017 WHO (c) 2020 Society for Endocrinology Published by Bioscientifica Ltd. Printed in Great Britain 
A

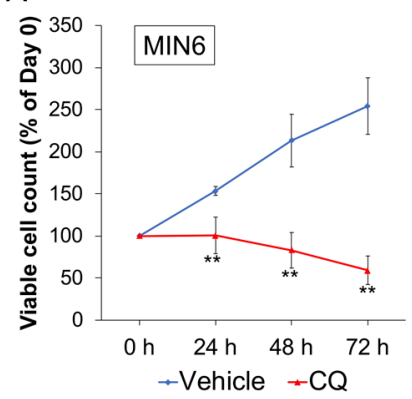

$\mathrm{D}$

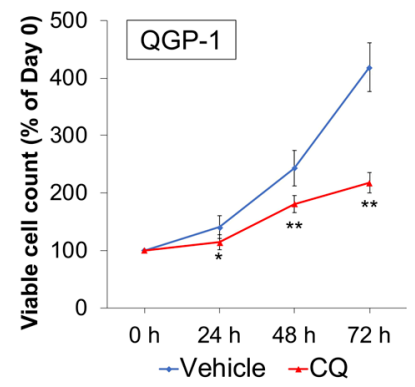

B

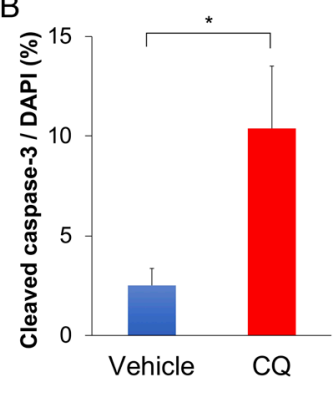

E

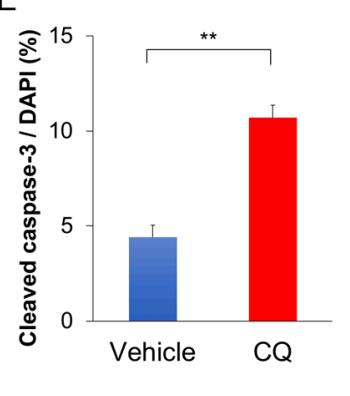

C

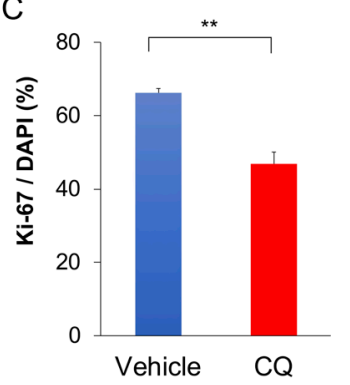

F

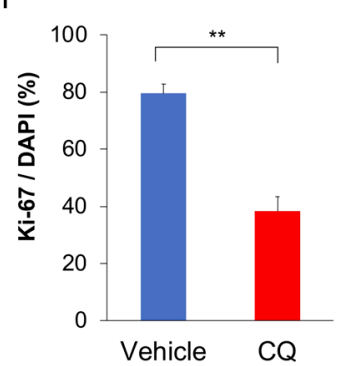

Figure 2

CQ shows inhibitory effects on PanNET cell lines. (A) Growth curve of MIN6 cells supplemented with $20 \mu \mathrm{M}$ of CQ or a vehicle ( $n=3$ in each group). (B and C) Immunofluorescent analysis of MIN6 cells receiving CQ treatment. Cleaved caspase-3-positive apoptotic cells are increased (B), and Ki-67-positive cells are decreased (C) ( $n=6 \mathrm{HPFs}$ in each group). (D, E, and F) Experiments with QGP-1 cells, done in triplicate manner. Data represent the mean \pm s.D. ${ }^{*} P<0.05 ; * \star P<0.01$, by unpaired $t$-test. classification. Therefore, we consider this mouse model to correspond to low-grade well-differentiated PanNETs in humans.

First, we intraperitoneally injected $\mathrm{HCQ}$, a derivative of CQ that is known to be more tolerable than CQ, into wild-type mice and evaluated the subsequent systematic autophagy-inhibiting activity. We found that $10 \mathrm{mg} / \mathrm{kg}$ of HCQ caused LC3-II accumulation in tissues including the ear and tail (Supplementary Fig. 1D). We then treated 18-month-old Men1+/4N3-8 mice with either HCQ (10 mg/kg/day) or saline every day for 3 weeks (Fig. 5A). Upon HCQ injection, the tumours in the HCQ group were significantly smaller than those in the saline-treated control group (Fig. 5B). The Ki-67 labelling index did not differ between the HCQ and saline groups (Fig. 5C), whereas TUNEL staining revealed significantly more apoptotic cells in the HCQ-treated tumours (Fig. 5D and E). The majority of tumour cells expressed CHOP following HCQ treatment (Fig. 5F). These findings indicate that HCQ treatment suppressed tumour growth by inducing apoptosis within PanNETs in Men1 $1^{+/ \Delta N 3-8}$ mice.

\section{Discussion}

The present study showed that the autophagy inhibitor CQ suppressed the growth of PanNEN tumour cells. In PanNEN cell lines, CQ suppressed cell proliferation and induced ER-stress-mediated apoptosis through the upregulation of the PERK-eIF2 $\alpha$-ATF4-CHOP pathway.
HCQ did not affect tumour incidence in $M e n 1^{+/ \Delta N 3-8}$ mice, but it did significantly suppress tumour growth in these mice by inducing apoptosis.

The present results are partially consistent with a previous report demonstrating CQ-induced apoptosis in a BON1 PanNEN cell line in vitro (Avniel-Polak et al. 2016). In terms of the efficacy of autophagy inhibitors in vivo, CQ showed additional effects over everolimus in the BON1 xenograft model; however, CQ treatment alone did not affect tumour growth or the induction of apoptosis (Avniel-Polak et al. 2018). Similarly, CQ alone did not show any apparent effect in the Rip1Tag2 mouse model, whereas the combination of CQ and sunitinib suppressed tumour growth in that same model (Wiedmer et al. 2017). Furthermore, we used a relatively low dose of HCQ $(10 \mathrm{mg} / \mathrm{kg})$, whereas $60 \mathrm{mg} / \mathrm{kg}$ of CQ was used in the BON1 xenograft model and $50 \mathrm{mg} / \mathrm{kg}$ of CQ was used in Rip1Tag2 mouse model. This dose setting was chosen according to the dose adopted in mouse studies on SLE (Gomez-Guzman et al. 2014), in which HCQ is widely used as an essential drug. The discrepancy between our study and previous studies might be derived from the differentiation status. In the BON-1 model and Rip1Tag2 mouse model, most of the PanNEN cell line conferred poorly differentiated tumours. In our in vitro model, MIN6 was derived from mouse insulinomas induced by insulin promoter-driven SV40 T antigens, which suppress p53 and RB activity. QGP-1 was established from human malignant somatostatinomas, but recently available genomic data have shown that QGP-1 has heterozygous 


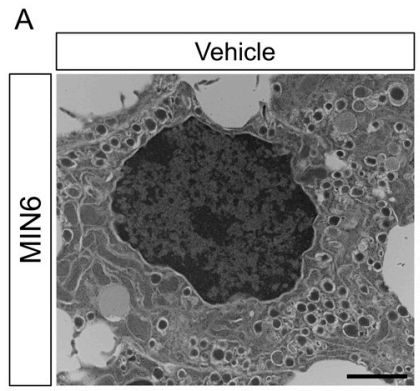

B

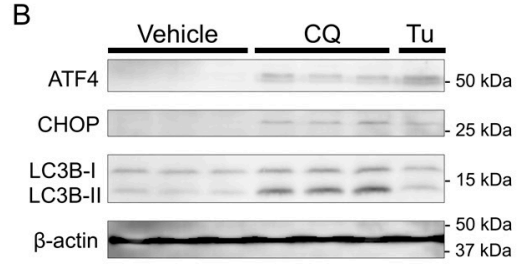

D

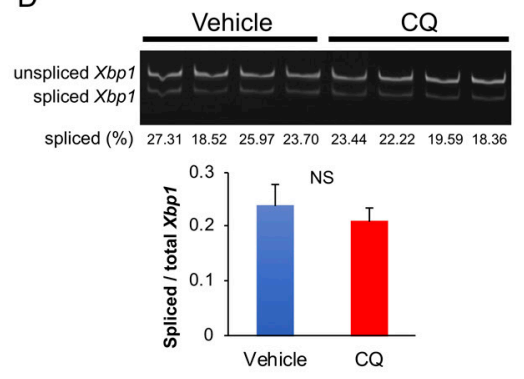

F

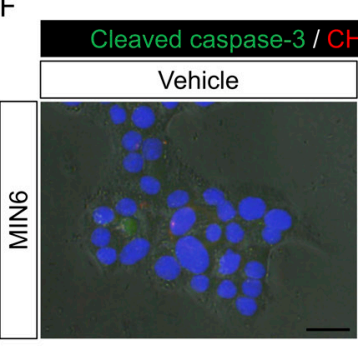

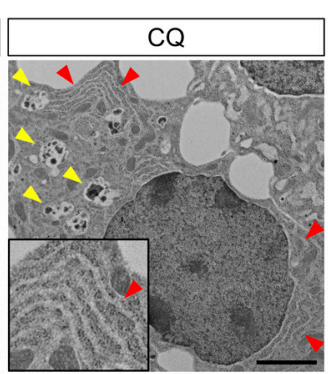

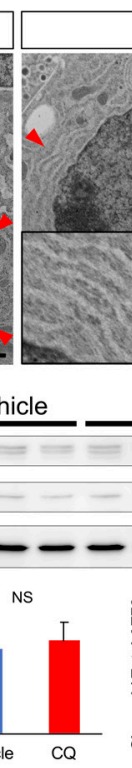

Tunicamycin

C

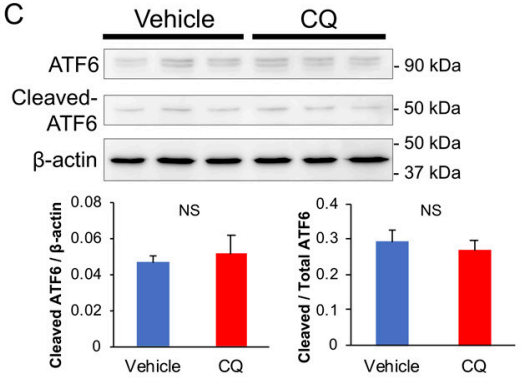

E
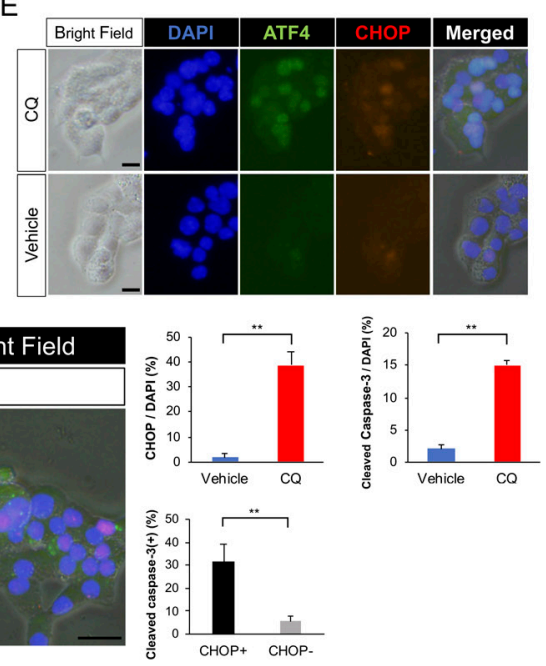

\section{Figure 3}

ER stress pathways in MIN6 cells treated with CQ. (A) TEM analyses of MIN6 cells supplemented with indicated reagents. CQ: $20 \mu \mathrm{M}$, tunicamycin: 1 $\mu \mathrm{g} / \mathrm{mL}$; Yellow arrowheads: autophagolysosomes; Red arrowheads: dilated ER. The insets show higher magnifications of dilated ER. Bar: $2 \mu \mathrm{m}$. (B) Induction of ATF4 and CHOP quantified by Western blot; $n=3$ in each group. Tu: tunicamycin $1 \mu \mathrm{g} / \mathrm{mL}$. (C) Cleavage of ATF6 quantified by Western blot; $n=3$ in each group. (D) Splicing of Xbp 1 mRNA quantified by qPCR; $n=4$ in each group. (E) Immunofluorescence of ATF4 and CHOP on MIN6 cells. Bar: $10 \mu \mathrm{m}$. (F) Immunofluorescence of $\mathrm{CHOP}$ and cleaved caspase- 3 on MIN6 cells; $n=4$ HPFs in each group. CQ: $20 \mu \mathrm{M}$. Data represent the mean \pm S.D. NS, not significant; $* P<0.05 ; * \star P<0.01$. Bar: $20 \mu \mathrm{m}$. mutations in KRAS (G12D) and TP53. Our current results showed that the Ki-67 positivity of the MIN6 and QGP-1 cells was $60-80 \%$, which was similar to those of BON1 cells and tumours of Rip1Tag2 mice in previous studies. In addition, BON1 cells have a mutation in NRAS and Rip1Tag2 mice were generated using SV40 Large T antigens in the insulin promoter. Taken together, the cell lines in our study and those of other studies are more likely to have characteristics of NEC rather than well-differentiated NETs. Compared to our results, in which HCQ alone at a relatively low dose showed inhibitory effects on tumours in Men1 $1^{+/ \Delta N 3-8}$ mice, the limited efficacy of CQ in previous reports may be explained by the poorly differentiation status and/or very high Ki-67 positivity in BON1 cells and tumours in Rip1Tag2 mice. These results suggest that HCQ monotherapy could be effective for tumours with low-grade lesions, while combination therapy might be preferable for higher-grade lesions in the application of clinical PanNEN treatments.

Previous studies have pointed out that autophagy inhibition induces apoptosis, but the underlying mechanisms have not yet been elucidated. Our findings showed that CQ/HCQ upregulated ER stress following the induction of apoptosis on CQ- or HCQ-treated PanNEN cells. In mice with genetically impaired autophagy caused by the pancreas-specific knockout of one of the autophagy-related genes (Atg7), acinar cells showed dilated ER (Antonucci et al. 2015), suggesting that the autophagy blockade induced ER stress. Those mice showed severe fibrosis and acinar-to-ductal metaplasia, acinar cell death, and regenerative proliferation in the pancreas. Our current results showed no impact on acinar cells; instead, they Printed in Great Britain 

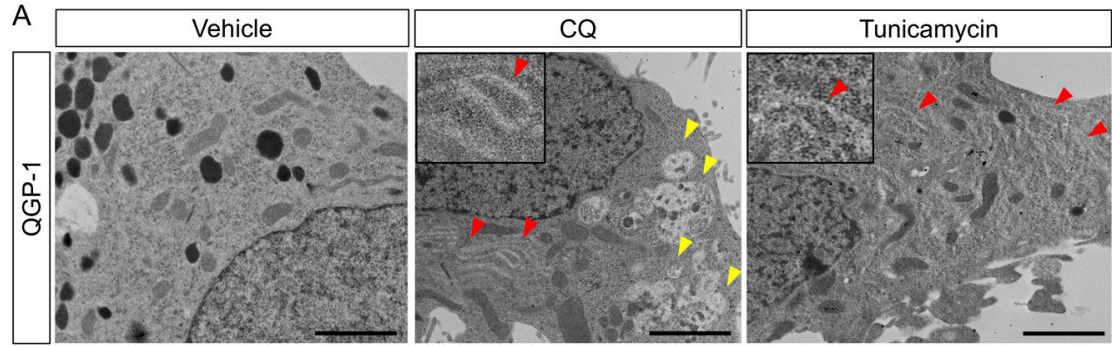

B

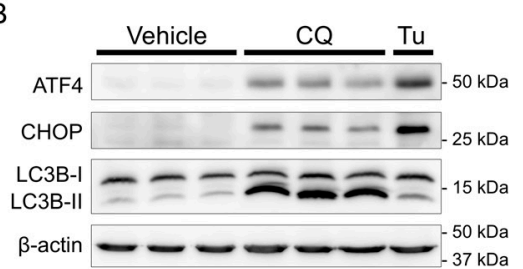

C

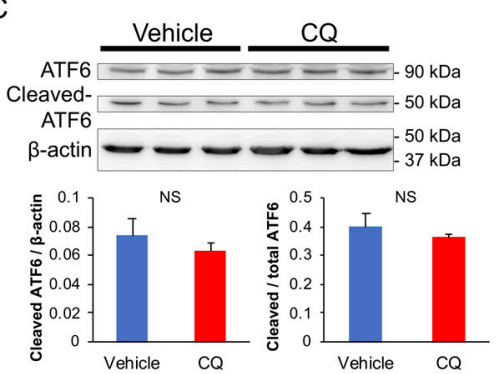

D

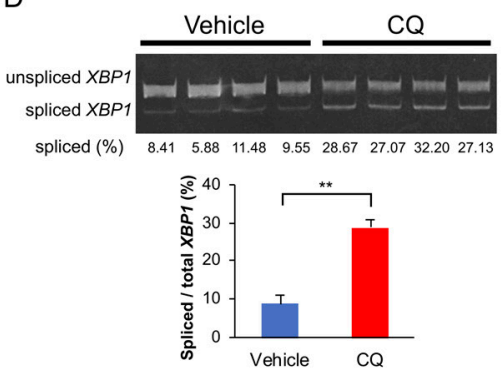

E

Cleaved caspase-3 / CHOP / DAPI / Bright Field Vehicle
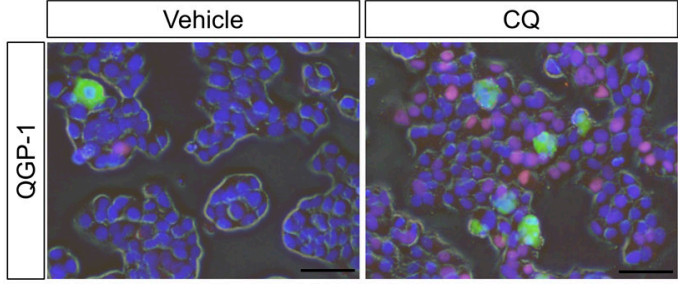

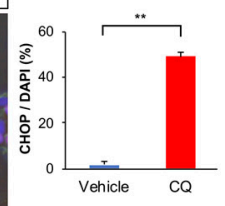

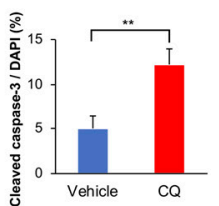

\section{Figure 4}

ER stress pathways in QGP-1 cells treated with CQ. (A) TEM analyses of QGP-1 cells supplemented with indicated reagents. CQ: $200 \mu \mathrm{M}$; tunicamycin: $10 \mu \mathrm{g} / \mathrm{mL}$; Yellow arrowheads:

autophagolysosomes; Red arrowheads: dilated ER. The insets show higher magnifications of dilated ER. Bar: $2 \mu \mathrm{m}$. (B) Induction of ATF4 and CHOP quantified by Western blot; $n=3$ in each group. CQ: $200 \mu \mathrm{M}$. Tu: tunicamycin $1 \mu \mathrm{g} / \mathrm{mL}$. (C) Cleavage of ATF6 quantified by Western blot; $n=3$ in each group. (D) Splicing of Xbp1 mRNA quantified by qPCR; $n=4$ in each group. (E) Immunofluorescence of CHOP and cleaved caspase-3 on QGP-1 cells; $n=6$ HPFs in each group. CQ: $200 \mu \mathrm{M}$. Data represent the mean \pm s.D. NS, not significant. $\star P<0.05 ; * * P<0.01$. Bar: $50 \mu \mathrm{m}$. showed tumour (or islet) cell-specific insults, suggesting that the autophagy blockade with CQ/HCQ affected islet cells rather than acinar cells. These findings suggest that the manifestation of cellular stress from an autophagy blockade could differ between cell types.

HCQ has some adverse effects, including retinopathy, neuropathy, and diarrhoea. A concern of applying HCQ in clinical trials is the development of diabetes; for example, we identified CHOP-positive cells among normal islets in the Men $1^{+/ \Delta N 3-8}$ mice. However, we found that the incidence of apoptosis in normal islets was lower than that among PanNEN tumour cells (data not shown). Moreover, a large cohort study showed that, in rheumatoid patients, those treated with HCQ exhibited a lower risk of diabetes because the anti-inflammatory effects of HCQ improved insulin resistance (Rempenault et al. 2018). Such findings suggest that HCQ-related insults to beta cells could be tolerable in the treatment of PanNEN patients.

Our study has several limitations that warrant consideration. First, we could not determine the impact of HCQ on the survival of PanNEN mice. When we bred Men1 ${ }^{+/ \Delta N 3-8}$ mice for 18 months, no significant difference in survival was evident between the Men1//AN3-8 mice and the wild-type control mice (data not shown). Therefore, we instead evaluated tumour size after a 21-day course of treatment and showed the inhibitory effects of HCQ. Second, we did not examine the synergic effects of mTOR inhibitors with HCQ on PanNENs, although mTOR inhibitors have been reported to accelerate autophagy (Klionsky et al. 2016). Further studies are necessary to clarify the impact of combination therapy.

In summary, we have demonstrated that autophagy inhibitors can induce apoptosis in PanNENs. 
A Men $1^{1 / \Delta N 3-8}$ mouse

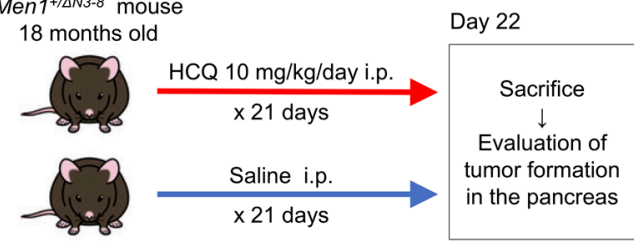

B

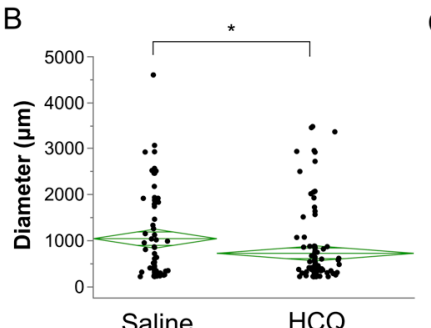

D

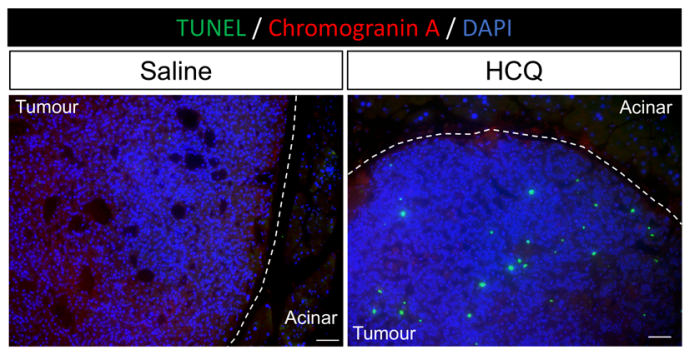

$\mathrm{F}$

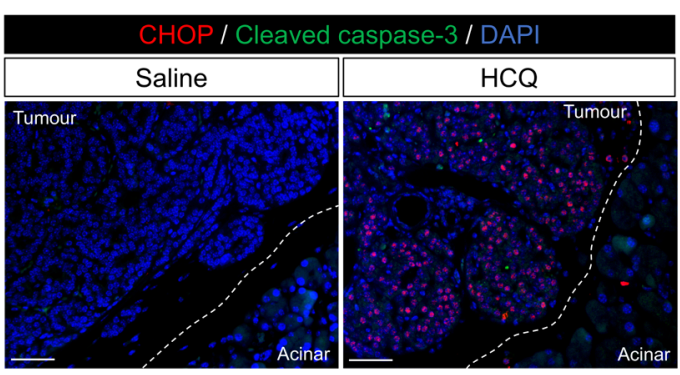

We also found that ER stress is one of the mechanisms underlying the induction of apoptosis in tumour cells. Our current results provide a new potential therapeutic approach for the treatment of PanNEN patients.

\section{Supplementary materials}

This is linked to the online version of the paper at https://doi.org/10.1530/ ERC-20-0028.

\section{Declaration of interest}

The authors declare that there is no conflict of interest that could be perceived as prejudicing the impartiality of the research reported.

\section{Funding}

This work was supported by the Japan Society for the Promotion of Science (USPS) KAKENHI Grant number JP18K08677.

\section{Figure 5}

HCQ treatment on Men 1+/LN3-8 mice results in reduced tumour size by inducing apoptosis. (A) In vivo experimental design; $n=6$ in each group. (B) Distribution of islet/tumour diameters. (C) Ki-67 labelling indexes of tumours from 18-month-old Men 1+/AN3-8 mice. Numbers of tumours, $n=10$ in the HCQ group and $n=12$ in the saline control group. ( $D$ and $E$ ) TUNEL staining of tumour sections. Number of TUNEL-positive cells is shown in (E); $n=6$ mice in each group. Bar: $50 \mu \mathrm{m}$. (F) Immunofluorescence of CHOP with cleaved caspase-3. Acinar: normal acinar cells; Tumour: tumour cells. Bar: $50 \mu \mathrm{m}$. Data represent the mean \pm s.D. NS, not significant; $* P<0.05$.

\section{Statement of ethics}

All the research was conducted ethically in accordance with the World Medical Association Declaration of Helsinki. The study design was approved by the institutional review board.

\section{Acknowledgements}

The authors thank Jun-ichi Miyazaki for kindly donating the MIN6 cells. The authors thank the staff of the Institute of Laboratory Animals at the Graduate School of Medicine, Kyoto University, for animal care. The authors thank Morito Sakikubo for the technical support with the ER stress analysis. The electron microscopy study was supported by Keiko OkamotoFuruta and Haruyasu Kohda (Division of Electron Microscopic Study, Center for Anatomical Studies, Graduate School of Medicine, Kyoto University).

\section{References}

Antonucci L, Fagman JB, Kim JY, Todoric J, Gukovsky I, Mackey M, Ellisman MH \& Karin M 2015 Basal autophagy maintains pancreatic acinar cell homeostasis and protein synthesis and prevents ER 
stress. PNAS 112 E6166-E6174. (https://doi.org/10.1073/ pnas.1519384112)

Avniel-Polak S, Leibowitz G, Riahi Y, Glaser B, Gross DJ \& GrozinskyGlasberg S 2016 Abrogation of autophagy by chloroquine alone or in combination with mTOR Inhibitors Induces apoptosis in neuroendocrine tumor cells. Neuroendocrinology 103 724-737. (https://doi.org/10.1159/000442589)

Avniel-Polak S, Leibowitz G, Doviner V, Gross DJ \& Grozinsky-Glasberg S 2018 Combining chloroquine with RAD001 inhibits tumor growth in a NEN mouse model. Endocrine-Related Cancer 25 677-686. (https://doi.org/10.1530/ERC-18-0121)

Bertolino P, Tong WM, Galendo D, Wang ZQ \& Zhang CX 2003 Heterozygous Men1 mutant mice develop a range of endocrine tumors mimicking multiple endocrine neoplasia type 1. Molecular Endocrinology 17 1880-1892. (https://doi.org/10.1210/me.2003-0154)

Chude CI \& Amaravadi RK 2017 Targeting autophagy in cancer: update on clinical trials and novel inhibitors. International Journal of Molecular Sciences 18 1279. (https://doi.org/10.3390/ijms18061279)

Crabtree JS, Scacheri PC, Ward JM, Garrett-Beal L, Emmert-Buck MR, Edgemon KA, Lorang D, Libutti SK, Chandrasekharappa SC, Marx SJ, et al. $2001 \mathrm{~A}$ mouse model of multiple endocrine neoplasia, type 1 , develops multiple endocrine tumors. PNAS 98 1118-1123. (https:// doi.org/10.1073/pnas.98.3.1118)

Dasari A, Shen C, Halperin D, Zhao B, Zhou S, Xu Y, Shih T \& Yao JC 2017 Trends in the incidence, prevalence, and survival outcomes in patients with neuroendocrine tumors in the United States. JAMA Oncology 3 1335-1342. (https://doi.org/10.1001/ jamaoncol.2017.0589)

Furuyama K, Kawaguchi Y, Akiyama H, Horiguchi M, Kodama S, Kuhara T, Hosokawa S, Elbahrawy A, Soeda T, Koizumi M, et al. 2011 Continuous cell supply from a Sox9-expressing progenitor zone in adult liver, exocrine pancreas and intestine. Nature Genetics $\mathbf{4 3}$ 34-41. (https://doi.org/10.1038/ng.722)

Gomez-Guzman M, Jimenez R, Romero M, Sanchez M, Zarzuelo MJ, Gomez-Morales M, O'Valle F, Lopez-Farre AJ, Algieri F, Galvez J, et al. 2014 Chronic hydroxychloroquine improves endothelial dysfunction and protects kidney in a mouse model of systemic lupus erythematosus. Hypertension 64 330-337. (https://doi.org/10.1161/ HYPERTENSIONAHA.114.03587)

Iguchi H, Hayashi I \& Kono A 1990 A somatostatin-secreting cell line established from a human pancreatic islet cell carcinoma (somatostatinoma): release experiment and immunohistochemical study. Cancer Research 50 3691-3693.

Kaku M, Nishiyama T, Yagawa K \& Abe M 1980 Establishment of a carcinoembryonic antigen-producing cell line from human pancreatic carcinoma. Gan 71 596-601.

Klionsky DJ \& Emr SD 2000 Autophagy as a regulated pathway of cellular degradation. Science 290 1717-1721. (https://doi. org/10.1126/science.290.5497.1717)

Klionsky DJ, Abdelmohsen K, Abe A, Abedin MJ, Abeliovich H, Acevedo Arozena A, Adachi H, Adams CM, Adams PD, Adeli K, et al. 2016 Guidelines for the use and interpretation of assays for monitoring autophagy (3rd edition). Autophagy 12 1-222. (https://doi.org/10.108 $0 / 15548627.2015 .1100356)$
Lee L, Igarashi H, Fujimori N, Hijioka M, Kawabe K, Oda Y, Jensen RT \& Ito T 2015 Long-term outcomes and prognostic factors in 78 Japanese patients with advanced pancreatic neuroendocrine neoplasms: a single-center retrospective study. Japanese Journal of Clinical Oncology 45 1131-1138. (https://doi.org/10.1093/jjco/ hyv143)

Loffler KA, Biondi CA, Gartside M, Waring P, Stark M, SerewkoAuret MM, Muller HK, Hayward NK \& Kay GF 2007 Broad tumor spectrum in a mouse model of multiple endocrine neoplasia type 1. International Journal of Cancer 120 259-267. (https://doi.org/10.1002/ ijc.22288)

Miyazaki J, Araki K, Yamato E, Ikegami H, Asano T, Shibasaki Y, Oka Y \& Yamamura K 1990 Establishment of a pancreatic beta cell line that retains glucose-inducible insulin secretion: special reference to expression of glucose transporter isoforms. Endocrinology 127 126-132. (https://doi.org/10.1210/endo-127-1-126)

Ogata M, Hino S, Saito A, Morikawa K, Kondo S, Kanemoto S, Murakami T, Taniguchi M, Tanii I, yoshinaga K, et al. 2006 Autophagy is activated for cell survival after endoplasmic reticulum stress. Molecular and Cellular Biology 26 9220-9231. (https://doi. org/10.1128/MCB.01453-06)

Rempenault C, Combe B, Barnetche T, Gaujoux-Viala C, Lukas C, Morel J \& Hua C 2018 Metabolic and cardiovascular benefits of hydroxychloroquine in patients with rheumatoid arthritis: a systematic review and meta-analysis. Annals of the Rheumatic Diseases 77 98-103. (https://doi.org/10.1136/ annrheumdis-2017-211836)

Ron D \& Walter P 2007 Signal integration in the endoplasmic reticulum unfolded protein response. Nature Reviews: Molecular Cell Biology $\mathbf{8}$ 519-529. (https://doi.org/10.1038/nrm2199)

Tabas I \& Ron D 2011 Integrating the mechanisms of apoptosis induced by endoplasmic reticulum stress. Nature Cell Biology 13 184-190. (https://doi.org/10.1038/ncb0311-184)

Wiedmer T, Blank A, Pantasis S, Normand L, Bill R, Krebs P, Tschan MP, Marinoni I \& Perren A 2017 Autophagy inhibition improves sunitinib efficacy in pancreatic neuroendocrine tumors via a lysosome-dependent mechanism. Molecular Cancer Therapeutics 16 2502-2515. (https://doi.org/10.1158/1535-7163.MCT-17-0136)

Xu R, Ji Z, Xu C \& Zhu J 2018 The clinical value of using chloroquine or hydroxychloroquine as autophagy inhibitors in the treatment of cancers: asystematic review and meta-analysis. Medicine $\mathbf{9 7}$ e12912. (https://doi.org/10.1097/MD.0000000000012912)

Yao JC, Hassan M, Phan A, Dagohoy C, Leary C, Mares JE, Abdalla EK, Fleming JB, Vauthey JN, Rashid A, et al. 2008 One hundred years after 'carcinoid': epidemiology of and prognostic factors for neuroendocrine tumors in 35,825 cases in the United States. Journal of Clinical Oncology 26 3063-3072. (https://doi.org/10.1200/ JCO.2007.15.4377)

Yoshida H, Okada T, Haze K, Yanagi H, Yura T, Negishi M \& Mori K 2000 ATF6 activated by proteolysis binds in the presence of NF-Y (CBF) directly to the cis-acting element responsible for the mammalian unfolded protein response. Molecular and Cellular Biology 20 6755-6767. (https://doi.org/10.1128/mcb.20.18.67556767.2000)

Received in final form 25 April 2020

Accepted 4 May 2020

Accepted Manuscript published online 5 May 2020 (c) 2020 Society for Endocrinology Published by Bioscientifica Ltd. Printed in Great Britain 